ÉGYPTE

monde arabe

\section{Égypte/Monde arabe}

22 | 1995

Géographies de l'Égypte 1

\title{
Les inondations de novembre 1994 en Égypte
}

Catastrophe naturelle... ou politique?

Habib Ayeb

\section{(2) OpenEdition}

Journals

Édition électronique

URL : https://journals.openedition.org/ema/612

DOI : $10.4000 /$ ema.612

ISSN : 2090-7273

Éditeur

CEDEJ - Centre d'études et de documentation économiques juridiques et sociales

Édition imprimée

Date de publication : 30 juin 1995

Pagination : 159-181

ISSN : 1110-5097

Référence électronique

Habib Ayeb, "Les inondations de novembre 1994 en Égypte », Égypte/Monde arabe [En ligne], 22 I

1995, mis en ligne le 08 juillet 2008, consulté le 07 juillet 2022. URL : http://journals.openedition.org/ ema/612 ; DOI : https://doi.org/10.4000/ema.612

Ce document a été généré automatiquement le 7 juillet 2022

Tous droits réservés 


\section{Les inondations de novembre 1994 en Égypte}

Catastrophe naturelle... ou politique?

\section{Habib Ayeb}

1 Le 12 octobre 1994, il a plu à Assouan pendant plus de dix heures sans interruption ${ }^{1}$. Le 2 novembre, en deux heures, le Caire a reçu $25 \mathrm{~mm}$ de pluie, dépassant de loin le dernier record (18 $\mathrm{mm}$ en 24 heures) enregistré le 29 novembre $1957^{2}$. En septembre 1994, avant d'atteindre l'Égypte, les inondations ont dévasté le nord du Soudan, provoquant des dégâts considérables. Ces pluies ont largement dépassé, par leur intensité, celles de 1988 et celles de 1946.

2 Les inondations de novembre ont marqué les mémoires, non seulement par leur importance exceptionnelle mais surtout parce qu'elles ont provoqué, directement et indirectement, des dégâts considérables : dans le seul village de Durunka (gouvernorat d'Asyut), elles ont été à l'origine d'un incendie qui a totalement ravagé l'agglomération où vivaient plus de 20.000 personnes, faisant, selon les chiffres officiels, plus de 500 morts, plusieurs centaines de blessés et plusieurs milliers de sans-abri. J'y reviendrai.

3 Ces inondations ont surtout révélé l'incapacité de l'État égyptien à prévoir ce genre de catastrophes et leurs conséquences et à venir efficacement au secours des populations sinistrées. Plus grave encore, elles ont révélé les défaillances des politiques d'aménagement du territoire et mis le doigt sur la « vétusté » d'une grande partie des infrastructures du pays.

Inondations et infrastructures

4 Outre l'état médiocre des voies de circulation entre les nombreux villages et villes de la vallée, qui explique en partie le retard dans l'acheminement des secours, il était clair que les modes d'extension du tissu urbain avaient depuis longtemps cessé de respecter les contraintes minimales de protection du bâti et des populations. Plusieurs faits sont à rappeler.

5 Les constructions s'élèvent le plus souvent en zone inondable, parfois même sur les lits d'oueds asséchés qui acheminaient vers le Nil les eaux de pluies tombées sur les collines 
enserrant la vallée. Souvent inondées, et subissant régulièrement les effets de la remontée de la nappe phréatique engendrée par la sur-irrigation, ces constructions, en cas de pluies exceptionnelles, se trouvent menacées par les flots qui dévalent la pente vers le fleuve.

6 L'interdiction de construire sur les terres agricoles, promulguée en 1982, a paradoxalement aggravé la situation. Dans l'impossibilité d'obtenir l'autorisation de construire sur ces terres, les familles, soumises à leur propre rythme d'accroissement naturel, finissent par bâtir illégalement en zone inondable - parfois même sur des parcelles agricoles. L'ampleur de ces «débordements » est telle que les autorités ne peuvent intervenir efficacement. Qu'elles en manifestent seulement l'intention, et les populations opposent une résistance farouche. Dans un village où les responsables locaux avaient tenté en vain de détruire des constructions non autorisées, les habitants avaient accepté de signer une déclaration dégageant les autorités de toute responsabilité en cas de catastrophe. Lourdement endommagé par les dernières inondations, ce village s'est vu rappeler cet engagement collectif.

7 Quant aux normes de sécurité concernant les constructions limitrophes des zones à risques, elles ne sont respectées ni par les habitants - mais les connaissent-ils ? - ni par les autorités locales. On peut voir telle usine, telle station service, tel dépôt de produits chimiques... enserrés dans un ensemble discontinu d'habitations, de magasins, d'ateliers, voire même d'écoles. Les exemples en sont nombreux mais celui de Durunka, avec son dépôt de pétrole, est tristement exemplaire. Nous en connaissons aujourd'hui les conséquences. L'on peut évidemment comprendre et même admettre que l'État se trouve dans l'incapacité d'empêcher ce genre de phénomènes; toutefois, les populations directement touchées ont tendance à percevoir, dans le silence des autorités, le double signe de leur incapacité, voire de leur complicité : en d'autres termes, leur responsabilité directe. L'État égyptien possède suffisamment de moyens pour prévoir les intempéries, avec des risques d'erreurs relativement faibles. Du reste, les services de la météorologie nationale avaient prévu les dernières inondations trois à quatre jours auparavant. Ces prévisions relativement précises ont été transmises à qui de droit et même publiées par la presse. Les responsables nationaux et locaux ne semblent pas en avoir tenu compte. Et quand la catastrophe a eu lieu, la «fatalité », le « destin » ont été érigés en justifications indiscutables.

Les fortes pluies en Égypte : un phénomène rare mais connu

8 A l'origine des pluies torrentielles qui ont ravagé une partie de l'Égypte, il y a eu un déplacement, vers le nord du FIT (front intertropical) qui a dépassé la ville d'Assouan. Conséquence immédiate de ce déplacement : la rencontre, au nord du Soudan et au sud de l'Égypte, des vents chauds et humides du sud et des vents froids venant du nord et du nord-ouest. Le contact entre les deux masses d'air a provoqué une remontée, vers la haute atmosphère, des vents chauds et humides, d'où une importante condensation qui s'est transformée en orages avec de très fortes chutes de pluies. D'après les services météorologiques de l'Égypte et du Soudan, cette perturbation s'est prolongée jusqu'au début du mois de novembre 1994, marqué par les inondations dont nous avons parlé.

Pour mieux comprendre ce qui s'est produit et éviter les polémiques, il n'est pas inutile de rappeler certains principes du fonctionnement des climats. Les vents venant du nord favorisent l'envoi vers le sud de masses d'air polaire froid. A l'opposé, les vents qui viennent du sud transportent des masses d'air chaud et parfois humide. Au contact des deux masses, l'air chaud, arrivant dans l'air froid, a tendance à s'élever rapidement au 
sein de celui-ci. La vapeur qu'il contient se condense alors, ce qui produit des nuages à développement vertical important, voire des orages. En temps "normal », le contact entre les deux masses d'air se produit au niveau de la zone de convergence intertropicale. Mais le cas «normal " n'est que très théorique puisque, soumis à des pressions d'origines et de forces diverses, le FIT se déplace, selon les saisons, vers le nord ou vers le sud.

Si, en hiver, le FIT a tendance à s'orienter vers le sud ou à rester sur place, l'été, il se déplace vers le nord, amenant derrière lui les pluies du Golfe de Guinée dans la partie ouest du Sahel, ou celles de l'océan Indien dans sa partie est. Ce phénomène touche le nord du Soudan et la Haute-Égypte entre les mois de septembre et novembre. Mais l'arrivée du FIT à cette latitude et la rencontre entre des masses d'air chaud et très humide et des masses d'air froid sont suffisamment rares pour que le phénomène soit inhabituel. En effet, au lieu de s'étendre sur l'Afrique de l'Est, les vapeurs d'eau qui se dégagent de l'océan indien sont le plus souvent détournées vers l'est par les vents alizés produisant les fameuses moussons, dont une partie tombe sur les zones montagneuses du Yémen. Dans ce cas, les vents chauds - mais débarrassés des vapeurs d'eau - qui arrivent sur le nord du Soudan et le sud de l'Égypte ne peuvent produire de la pluie mais seulement des perturbations qui marquent annuellement l'automne égyptien. En effet, il ne pleut que si - et seulement si - «deux conditions sont réalisées : l'arrangement des courants atmosphériques horizontaux est tel qu'il y a des arrivées d'air humide - on dit parfois "des advections de vapeur d'eau »; il existe des ascendances de l'air humide, qui provoquent son refroidissement et donc des condensations de cette vapeur d'eau. $»^{3}$

Tableau $1: 25$ ans d'inondations

\begin{tabular}{|c|c|c|}
\hline Dates & Lieux & Bilan \\
\hline Octobre 69 & $\begin{array}{l}\text { Giza, Bani Swayf, } \\
\text { Assouan }\end{array}$ & 162 maisons détruites et 169 personnes sans abri \\
\hline $\begin{array}{l}\text { Décembre } \\
70\end{array}$ & $\begin{array}{l}\text { Damiette, Ra's al- } \\
\text { Bar }\end{array}$ & $\begin{array}{l}\text { Paralysie de la circulation, coupures de courant et de lignes } \\
\text { téléphoniques }\end{array}$ \\
\hline Janvier 71 & $\begin{array}{l}\text { Gharbiyya (Delta), } \\
\text { Ibis, Alexandrie }\end{array}$ & 18 morts, 160 maisons détruites et 200 familles sans abri \\
\hline Février 75 & Suhag & $\begin{array}{l}\text { Destruction complète du village de Nazlat al-Qâdi, près de } \\
\text { Suhag. } 20 \text { victimes, destruction de } 4.000 \text { logements à Minya }\end{array}$ \\
\hline 1981 & Sinaï et mer Rouge & $\begin{array}{l}100.000 \text { personnes privées de toit à al-Arîsh, Routes détruites } \\
\text { (Ste-Catherine, Nuwayba, Golfe de Suez) }\end{array}$ \\
\hline 1982 & Sinaï & 1.500 personnes sans abri \\
\hline $\begin{array}{l}\text { Décembre } \\
85\end{array}$ & Asyut & Destruction du village de 'Arab \\
\hline
\end{tabular}




\begin{tabular}{|c|c|c|}
\hline $\begin{array}{l}\text { Novembre } \\
87\end{array}$ & Sinaï, Qena, Assouan & Dégâts importants \\
\hline 1988 & Sinaï & Routes coupées \\
\hline Mai 89 & Qena & 15 morts, 400 logements détruits \\
\hline Octobre 89 & Côtes nord & Routes coupées entre Alexandrie et Matrûh \\
\hline Mars 90 & Sinaï & $\begin{array}{l}\text { Routes du Sud-Sinaï coupées. Accident d'autobus: tous les } \\
\text { passagers trouvent la mort }\end{array}$ \\
\hline Octobre 93 & Halaïb et mer Rouge & Dégâts matériels importants \\
\hline Janvier 94 & Sinaï & $\begin{array}{l}\text { Des torrents de boue traversent le sud du Sinaï à une vitesse } \\
\text { de } 150 \mathrm{~km} / \mathrm{h} \text { sans faire de victimes; dégâts matériels très } \\
\text { importants }\end{array}$ \\
\hline $\begin{array}{l}\text { Oct./nov. } \\
94\end{array}$ & Haute-Égypte & ( $C f$. bilan détaillé dans le présent article). \\
\hline
\end{tabular}

Source : al-Ahrâm Hebdo, 09-15/11/94.

11 Ainsi, même exceptionnelles par leur intensité et leur périodicité, les pluies du 2 novembre n'avaient rien d'extraordinaire et n'étaient pas annonciatrices d'un changement de climat, comme cela a pu être énoncé. En général, l'automne se caractérise par des perturbations météorologiques, même si elles ne donnent pas toujours naissance à des précipitations importantes. Celles de l'automne 1994 ne sont que les dernières d'une série relativement régulière, dont la génération des quarante ans pourrait témoigner.

12 Certes, le nombre de victimes enregistré cette année est particulièrement élevé ; mais il faut enlever, du chiffre global, les 500 morts officiellement recensés dans l'incendie de Durunka qui, tout en relevant des conséquences indirectes de ces intempéries, sont d'abord les sinistrés d'un système d'aménagement du territoire conçu sur le principe du moindre investissement. Il ne s'agit pas ici de dire que l'État égyptien se désengage de son rôle d'aménageur - cela reviendrait en quelque sorte à ignorer l'histoire et la géographie de ce pays - mais de souligner certaines failles dans son système de gestion de l'espace. La première de ces failles me semble relever de la difficulté de mobilisation de la mémoire collective et de l'histoire récente pour faire face aux événements « naturels » et, en particulier, aux inondations. En effet, comme je le soulignais plus haut, les Égyptiens n'ignorent pas ce phénomène, mais on a le sentiment qu'il s'agit à chaque fois d'une situation tout à fait nouvelle.

Des inondations pas si anciennes... et pourtant oubliées

13 Je me limiterai ici à rappeler (tableau 1) les inondations les plus importantes qui ont touché l'Égypte au cours des 25 dernières années. Soulignons dès à présent que certaines avaient touché les mêmes régions et engendré des dégâts comparables. Déjà, en 1975, des inondations similaires avaient détruit les villages de Shaykh Tama, Bani- 
Hasan al-Shuruq, Dir al-Barsha et Nazlat-al-Barsha ${ }^{4}$. Reste à rappeler que, bien entendu, toutes les pluies n'ont pas les mêmes origines que le phénomène développé ci-dessus.

Le haut barrage hors de cause

14 Lorsqu'une catastrophe naturelle se produit en Égypte - sécheresse, inondations, tremblement de terre - maints regards se tournent vers le haut barrage d'Assouan et le lac Nasser, soupçonnés d'être à l'origine de tout ce qui se produit en aval. En particulier, on voudrait faire croire que le haut barrage est à l'origine d'un changement radical du climat égyptien, qui serait lui-même à l'origine des pluies exceptionnelles.

Cette thèse, totalement infondée à mon sens, suscite un certain nombre de remarques. D'une part, il est scientifiquement impossible de conclure à un changement définitif du climat en partant d'une période d'observation qui ne dépasse pas les quarante ans - le lac Nasser n'existe que depuis 1964. L'histoire des climats indique en effet des cycles relativement réguliers où alternent des périodes de haute et de basse pluviométrie. Ces périodes, pouvant aller de quelques années à quelques dizaines d'années, sont souvent marquées par des « pics » trop forts ou au contraire trop faibles. Aussi, pour étudier les changements climatiques, faut-il envisager une période d'observation très longue couvrant au minimum une centaine d'années afin de tenir compte des cycles centenaires, c'est-à-dire de phénomènes climatiques qui reviennent tous les cent ans en moyenne. Ni les dernières inondations, ni les périodes de sécheresse qui ont sévi pendant les années 80 ne peuvent indiquer un changement irréversible du climat de la région.

16 Si le volume de la masse d'eau accumulée dans le lac Nasser pouvait expliquer l'augmentation, du reste très modeste, du taux d'humidité dans la région d'Assouan, et plus particulièrement autour du lac, elle ne peut être à l'origine de pluies torrentielles qui se produisent à plusieurs centaines de kilomètres de là, tant au sud qu'au nord de la grande digue.

17 D'ailleurs, durant les années 80, le niveau du lac avait connu une baisse importante sans que cela empêche l'arrivée de fortes pluies, qui ont laissé des dégâts considérables tant au Soudan qu'en Égypte. Les inondations qui ont frappé le Soudan pendant l'été 1988 se sont justement produites à un moment où le lac Nasser avait atteint son niveau le plus bas depuis la mise en eau du barrage en 1964. De même, comme le rappelle Mahmûd alJamal, chef de l'Autorité générale du haut barrage d'Assouan, "aucune pluie significative n'a été enregistrée en 1978 quand le lac était arrivé quasiment à son maximum, atteignant la cote de 177,48 mètres $"^{5}$.

Le tableau suivant montre clairement que les pluies torrentielles sont loin d'être un phénomène récent et lié au haut barrage, mais qu'au contraire l'Égypte l'a toujours connu, du moins depuis le début du siècle, période pour laquelle nous disposons d'observations et de témoignages fiables : 
Tableau 2: Les plus fortes inondations en Égypte depuis le début du siècle

\begin{tabular}{|l|c|cc|}
\hline \multicolumn{1}{|c|}{ Lieux } & Avant le haut barrage & \multicolumn{2}{c|}{ Après le haut barrage } \\
\hline Assouan & $1928-1936-1947-1960$ & \multicolumn{2}{c|}{$1970-1971-1975-1979$} \\
Qena & 1945 & $1967-1970$ & 1979 \\
Suhag & 1950 & & $1975-1979$ \\
Asyut & & 1975 \\
Minya & & 1965 & 1975 \\
Bani Swayf & & & 1975 \\
\hline
\end{tabular}

Source : Comité technique du Syndicat des ingénieurs publié par le journal al-Wafd du 15/11/94. Ces inondations ne concernent pas celles qui étaient provoquées par le Nil avant la mise en eau du barrage en 1964

Toutefois, même si l'on acceptait l'hypothèse, tout à fait théorique et invérifiable à court terme, d'une influence du lac Nasser sur le climat égyptien, il serait mal venu de ne pas rappeler le rôle protecteur que le haut barrage n'a cessé de remplir contre la crue du Nil et la sécheresse.

Limite des prévisions météorologiques

Les prévisions météorologiques les plus fiables - et celles des services égyptiens le sont parfaitement - sont-elles en mesure d'éviter ce genre de catastrophes? Assurément non puisque, comme nous le verrons plus loin, le problème n'est pas tant le caractère inattendu de ces inondations qu'une politique d'aménagement du territoire qui ne tient aucun compte des risques naturels. Du reste, l'Égypte n'est pas le seul pays à souffrir de cette défaillance. Il suffit de rappeler les inondations qui ont frappé plusieurs régions françaises ces dernières années, les plus récentes ayant touché, en janvier-février 1995, 43 départements. Tous les moyens techniques dont disposent les autorités françaises, alliés à diverses formes de compétences administratives, politiques et scientifiques, n'ont pu empêcher des dégâts considérables et des pertes humaines (18 morts). Ce qu'il faut souligner, si l'on compare ces événements avec ce qui s'est passé en Égypte, c'est le fait que la quasi-totalité des destructions a été enregistrée là où il y avait des constructions, souvent tout à fait légales, en zone inondable. La comparaison s'arrête là ; les deux États ne disposent pas des mêmes moyens d'intervention, de secours et, surtout, de protection des zones et des populations exposées. C'est cette différence de moyens, mais aussi de politiques d'aménagement, qui fait que deux catastrophes comparables laissent en général un bilan beaucoup plus lourd dans un État en voie de développement.

Pour en revenir à la capacité prévisionnelle des services météorologiques, il faut noter qu'ils avaient prévu les inondations et prévenu le gouvernement pratiquement un mois et demi avant les événements. Selon le rapport remis aux autorités, le risque d'inondation était plus fort que jamais. Le rapport précisait d'ailleurs les lieux menacés : la chaîne des montagnes de la mer Rouge et la vallée du Nil en Haute-Égypte, et particulièrement les deux gouvernorats de Sohag et de Qena ${ }^{6}$. Mais ces avertissements ne reposaient en vérité que sur de simples hypothèses. Cela ne suffisait pas pour envisager, chez les responsables politiques et ceux de la protection civile, des réactions rapides et systématiques. 

météorologie, " pour ce qui est des prévisions, nous nous limitons à trois jours, au-delà desquels les risques d'erreurs vont croissant $»^{7}$. Il précise que s'il est «impossible d'estimer à l'avance les quantités de pluies attendues, on peut cependant dire, trois jours avant, si les pluies seront fortes, moyennes ou faibles. »

. 'Abd al-Rahmân, dont les services ont été accusés de négligence, n'a pas hésité à mettre les autorités du pays devant leurs responsabilités : « Nous avons prévu les pluies du 2 novembre et avons envoyé, le dimanche 30/10/94, un avertissement qui a été publié dans tous les journaux en date du 31/10. En effet, les pluies annoncées sont arrivées le 02/11/94. $»^{8}$

BilanImportance et extension des dégâts national égyptien, et plus particulièrement la vallée du Nil jusqu'au nord du Soudan, a été plus ou moins gravement touché par les conséquences directes et/ou indirectes des pluies. Dans le nord du Soudan, les inondations ont gravement endommagé le port de Wâdi Halfa, provoquant l'interruption de la navigation sur le lac Nasser ${ }^{9}$. En aval du haut barrage, de nombreux villages ont été gravement sinistrés, la navigation a été suspendue entre Assouan et Louxor et les touristes en croisière ont été ramenés à terre ${ }^{10}$. Enfin, dans le nord du pays, les routes allant vers la Libye et le nord du Sinaï ont été fermées à la circulation pendant plusieurs jours ${ }^{11}$.

A la mi-novembre 1994, les recensements publiés par la presse gouvernementale (qu'on peut imaginer légèrement sous-estimés) sont impressionnants : 230 millions $\mathrm{de}^{3}$ d'eau sont tombés sur la Haute-Égypte, dont 120 millions sur le seul gouvernorat de Sohag, touchant 35 villages sur une distance de $200 \mathrm{~km}$ entre la ville de Sohag et le district de Sagilta. 70 millions de $\mathrm{m}^{3}$ ont touché les villages de la région de Dâr al-Salâm, qui a le plus souffert ${ }^{12}$. A Qom Ombo, les inondations ont détruit cinq villages et endommagé, sur pied, toute la récolte de dattes de cette année ${ }^{13}$. Une semaine plus tard, les chiffres seront révisés à la hausse : on parle désormais de 55 villages touchés en Haute-Égypte, selon les chiffres du Ministère des Ressources hydrauliques ${ }^{14}$.

La catastrophe de Durunka

C'est avec l'incendie de Durunka, le soir du 2 novembre, que la catastrophe a pris une ampleur sans précédent. Quelque 500 personnes ont péri et plusieurs centaines de familles rescapées se sont retrouvées sans abri. Quant à la localité de Durunka ellemême, elle a été presque entièrement détruite.

Durunka, situé à quelques kilomètres au sud-ouest d'Asyut, sur la rive gauche du Nil, était par plusieurs aspects semblable à la plupart des villages qui bordent le fleuve entre Giza et Assouan. Sa position géographique, comme celle d'autres localités, n'était pas le fait du hasard: c'est, paradoxalement, pour échapper à la crue du Nil que ces villages sont nés sur la lisière des terres agricoles, qui étaient annuellement plus ou moins gravement touchées par la crue du fleuve. La zone agricole, antérieurement inondable et actuellement protégée grâce au haut barrage, qui a définitivement mis fin à la crue, est aujourd'hui entièrement cultivée, d'où l'impossibilité, pour les habitants de ces villages, de s'étendre vers le fleuve. Ainsi les constructions, légales ou non, se sont-elles progressivement avancées sur l'espace compris entre les terres irriguées et les collines avoisinantes, empiétant souvent sur les lits des oueds et s'exposant aux torrents que provoquent occasionnellement les pluies. 
Situé, donc, à l'extrémité ouest des terres cultivées de la vallée, ce village est dominé à l'ouest par les hautes collines qui bordent ce segment de la vallée et a empêché son extension vers l'ouest. La pente relativement importante et l'absence, d'une part, de couverture végétale et, d'autre part, de régularité pluviométrique, ce qui rend le sol compact et donc très peu perméable, font que la quasi-totalité des pluies qui tombent sur ces collines dévalent très rapidement la pente en emportant des quantités considérables de boue. Arrivées au pied des collines, ces eaux boueuses s'engouffrent dans les lits des oueds avant d'atteindre le Nil ${ }^{15}$. Les eaux excédentaires débordent des oueds et fuient vers l'aval à une rapidité plus ou moins importante selon les obstacles rencontrés. C'est précisément sur ces zones inondables qu'il conviendrait d'empêcher toute extension de l'urbanisation. A Durunka, non seulement on n'a rien fait de tel, mais on a construit, sur une partie de cette zone inondable, un dépôt de stockage de pétrole et une ligne de chemin de fer reliant ce dépôt à la ville d'Asyut ${ }^{16}$.

Tableau 3 : Evolution de la population de Durunka et d'Asyut de 1882 à 1994

\begin{tabular}{|c|c|c|c|c|c|c|c|c|c|c|c|c|c|}
\hline Source & Année & Durun & & & & & & & & Asyu & & & \\
\hline \multirow[t]{2}{*}{$\mathrm{RP}$} & \multirow[t]{2}{*}{1882} & (1) & $(2)$ & (3) & (4) & (5) & \multicolumn{2}{|c|}{ Agglomér. } & \multicolumn{2}{|c|}{ dont ville } & \multicolumn{2}{|c|}{ dont banlieue } & \\
\hline & & 5.170 & 4.629 & - & 54 & 541 & 31.575 & \multicolumn{3}{|c|}{31.575} & \multicolumn{2}{|l|}{-} & \\
\hline $\mathrm{RP}$ & 1897 & 6.460 & \multicolumn{2}{|c|}{5.722} & \multicolumn{2}{|c|}{-} & 738 & \multicolumn{2}{|c|}{738} & \multicolumn{2}{|c|}{42.078} & 42.078 & - \\
\hline $\mathrm{RP}$ & 1907 & 6.973 & \multicolumn{2}{|c|}{6.973} & \multicolumn{2}{|c|}{-} & - & \multicolumn{2}{|l|}{ - } & \multicolumn{2}{|c|}{39.442} & 39.442 & - \\
\hline $\mathrm{RP}$ & 1917 & 7.679 & \multicolumn{2}{|c|}{7.679} & \multicolumn{2}{|c|}{-} & - & \multicolumn{2}{|l|}{ - } & \multicolumn{2}{|c|}{51.431} & 51.431 & - \\
\hline $\mathrm{RP}$ & 1927 & 8.333 & \multicolumn{2}{|c|}{8.333} & \multicolumn{2}{|c|}{7.130} & - & \multicolumn{2}{|l|}{ - } & \multicolumn{2}{|c|}{57.134} & 57.134 & - \\
\hline $\mathrm{RP}$ & 1937 & 9.846 & \multicolumn{2}{|c|}{9.846} & \multicolumn{2}{|c|}{-} & - & \multicolumn{2}{|l|}{ - } & \multicolumn{2}{|c|}{60.338} & 60.338 & - \\
\hline $\mathrm{RP}$ & 1947 & 10.593 & \multicolumn{2}{|c|}{10.593} & \multicolumn{2}{|c|}{-} & - & \multicolumn{2}{|l|}{ - } & \multicolumn{2}{|c|}{92.758} & 90.333 & 2.425 \\
\hline $\mathrm{RP}$ & 1960 & 14.694 & \multicolumn{2}{|c|}{12.553} & \multicolumn{2}{|c|}{-} & 2.141 & 2.1 & 141 & 129. & 799 & 127.485 & 2.314 \\
\hline $\mathrm{RP}$ & 1966 & 16.752 & 14. & & & .497 & 2.255 & 2.2 & 255 & 156. & 321 & 153.956 & 2.365 \\
\hline $\mathrm{RP}$ & 1976 & 21.832 & 19.? & 742 & & .742 & 2.090 & 2.0 & 090 & 216. & 900 & 213.751 & 3.149 \\
\hline $\mathrm{RP}$ & 1986 & 29.197 & 26. & 722 & & 610 & 2.475 & 2.4 & 473 & 287. & 461 & 273.191 & 14.270 \\
\hline Estim. & 1994 & 35.000 & 32.2 & 200 & - & & - & - & & 343. & 000 & 320.000 & 23.000 \\
\hline
\end{tabular}




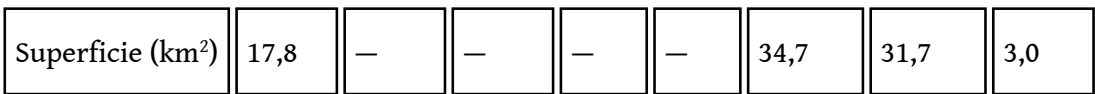

(1) Durunka et Dayr Durunka.

(2) Durunka : population totale.

(3) Durunka : population agglomérée.

(4) Dayr Durunka : population totale.

(5) Dayr Durunka : population agglomérée.

Source OUCC, Base de données «EGIPTE »

29 C'est, en effet, immédiatement en contrebas de la colline - un endroit protégé des regards indiscrets -, que l'armée égyptienne avait décidé, au début des années 50, d'installer des réservoirs stratégiques de produits pétroliers destinés à être utilisés au cas où d'autres lieux de stockage plus exposés seraient attaqués. Une ligne de chemin de fer à une voie, construite sur une simple digue en terre battue, permettait de transporter le pétrole.

$\mathrm{Au}$ lendemain de la signature des accords de paix avec Israël, l'armée égyptienne a vendu ces réservoirs à une coopérative de carburants ${ }^{17}$ et le passage d'un statut purement militaire à un statut civil s'est accompagné d'une relâche des mesures de sécurité. L'interdiction étant donc implicitement levée, les populations en manque d'espace ont commencé à grignoter les terrains qui constituaient à l'époque une sorte de périmètre de sécurité infranchissable. Avant d'être complètement ravagé par les flammes, le village avait fini par enserrer totalement les réservoirs et tous les terrains libres le long de la voie chemin de fer. Cette extension incontrôlée du village s'est donc opérée sur un espace doublement dangereux : une zone inondable et aussi, peut-être surtout, une zone sous menace permanente d'explosion ou d'incendie. Deux risques que ni les habitants ni les autorités n'avaient apparemment mesurés à leur juste valeur.

31 Cette extension du tissu urbain s'explique par une très forte croissance de la population de l'agglomération, qui est passée de 14.497 habitants en 1966 à 26.722 en 1986 et 32.200 en 1994. La population regroupée de Durunka et de Dayr Durunka est passée, pour les mêmes périodes, de 16.752 habitants à 29.197 et 35.000 . Face à ce doublement de la population en moins de trente ans ${ }^{18}$, on comprend mieux l'ampleur de l'extension du bâti (Voir tableau 3 ci-contre, et cartes pages suivantes).

Rappelons la manière dont la catastrophe s'est produite. Au cours de la nuit du 2 novembre, qui a vu des pluies exceptionnellement fortes s'abattre sur la région, les torrents chargés de grandes quantités de boue arrachée aux collines et aux lits des oueds envahissent le village. Les eaux provoquent un glissement de terrain qui ébranle les fondations de la voie de chemin de fer. A ce moment, le train qui passait, chargé de pétrole, déraille. Le choc provoque des étincelles qui entraînent l'explosion des citernes. Le feu atteint rapidement deux cuves du dépôt, provoquant une nouvelle série $\mathrm{d}^{\prime}$ explosions ${ }^{19}$. Le pétrole en flammes se répand alors en nappe sur les torrents, qui dévalent vers le village et propagent l'incendie ${ }^{20}$. Les habitants, surpris dans leur sommeil, sont pris au piège ${ }^{21}$. 
Le site de Durunka

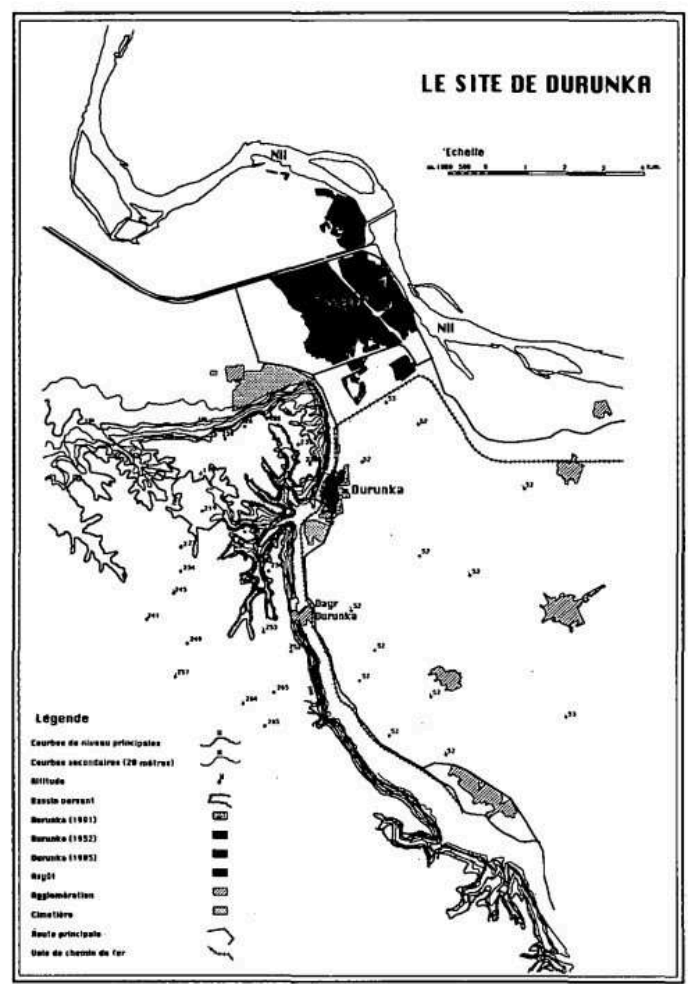

Durunka et Dayr Durunka

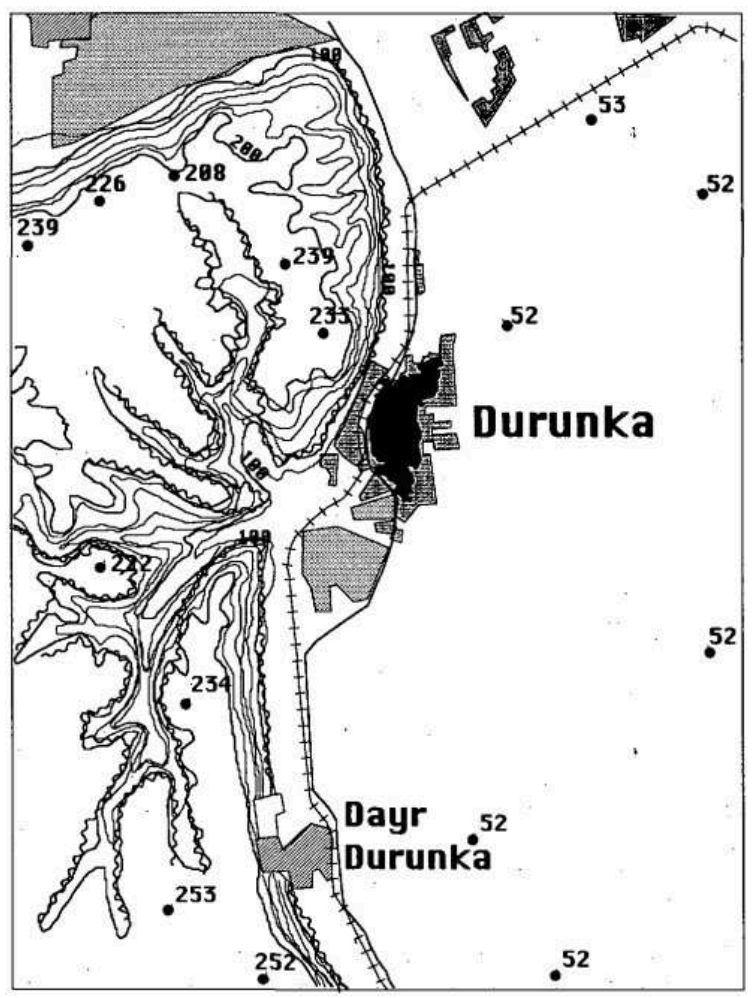

33 La responsabilité des autorités est démontrée dans le rapport du Comité d'enquête du Syndicat des ingénieurs égyptiens, qui précise que «le site n'était pas équipé de 
moyens automatiques de prévention et de lutte contre l'incendie $»^{22}$. Il s'agit d'un système qui se déclenche, grâce à un thermostat, lors d'une forte hausse de température, et qui permet de circonscrire le feu dans un espace limité. A Durunka, libéré de toute contrainte, le feu n'a pu être circonscrit, d'autant que les pompiers et les équipes de sauvetages ne sont intervenus que plusieurs heures après le début de l'incendie ${ }^{23}$.

Mais l'on ne peut se contenter de souligner, même à raison, la responsabilité des autorités à tous les niveaux de l'échelle administrative et politique. Les populations locales assument une part de responsabilité, même si l'on peut leur trouver des circonstances atténuantes (comment peut-on, en effet, reprocher à des paysans peu fortunés de construire - même au mépris des risques et des lois en vigueur, que les autorités locales elles-mêmes ne respectent pas - une maison modeste qui leur permettra d'alléger la pression du nombre dans la demeure familiale ?). M. Sabû alChatt Taha, directeur de la planification régionale au Ministère de la Construction, estime que «la population locale a, de fait, une part de responsabilité. Les dépôts de carburant du village de Durunka existant depuis [longtemps], l'erreur réside dans l'avancée anarchique des habitations vers ces dépôts. Les localités devaient raser ces quartiers. $»^{24}$

Le quotidien al-Ahrâm, tout en désignant «l'habitat informel » comme responsable du drame, relativise le constat en soulignant que derrière le non-respect des lois et des règles fondamentales de l'aménagement du territoire, on trouve une logique de « laisser-faire » qui s'applique à l'ensemble du territoire national : «L'habitat informel est effectivement à l'origine du drame, mais les habitants de Durunka ont fait comme ceux de tous les villages ruraux d'Égypte, où ce mode de construction est la règle. Pourquoi les habitants de Durunka auraient-ils respecté les règlements? Ils ne sont ni plus diplômés ni mieux informés que les autres. $»^{25}$ «Faut-il accuser les habitants de tous ces villages, ou l'accusation doit-elle se limiter aux 500 morts de Durunka?» s'interroge le chroniqueur d'al-Ahrâm ${ }^{26}$.

Le rapport du comité d'enquête du Syndicat des ingénieurs désigne, derrière ce laisseraller, les relations de clientélisme qu'entretiennent les responsables politiques avec les populations et précise que "les constructions non réglementaires à côté du dépôt se sont faites sous la protection et avec l'accord d'un membre de l'Assemblée du Peuple $\aleph^{27}$. Et comme pour apporter la preuve de cette complicité, le journal al-Ahrâm rappelle que tout le village de Durunka, dont les zones d'habitat informel, était rattaché au réseau électrique et avait l'eau courante ${ }^{28}$, ce qui donne à cette zone une semilégalité.

Quant à l'hebdomadaire Rûz al-Yûsuf, il souligne, à sa manière, les responsabilités des uns et des autres : «Si les citoyens sont responsables, les autorités locales le sont bien davantage : elles les ont laissé construire sur un terrain que l'on savait être un point de convergence des eaux en temps de pluie. Cela nous invite à prendre conscience d'autres dangers dus à la négligence, comme ces tours, au Caire, démunies de moyens de lutte contre les incendies... »

38 L'ensemble des reportages, prises de position, aveux et explications plus ou moins objectives montre, s'il en était besoin, la complexité de la situation : si les inondations relèvent d'un phénomène naturel, les catastrophes qui en découlent relèvent quasi exclusivement des politiques de gestion de l'espace. Toutefois, si l'homme produit l'espace, il n'en assume pas l'exclusive responsabilité. De multiples contraintes 
interviennent d'une manière décisive pour remodeler l'espace, allant jusqu'à lui imprimer une marque inattendue ou imprévisible.

39 Autrement dit, il est nécessaire d'opérer un renversement des rôles et des responsabilités pour mieux souligner celle du détenteur du pouvoir, qu'il soit régional ou national. Certes, personne ne peut ignorer le non-respect des règles d'aménagement par les populations concernées, ni l'importance quantitative des constructions dites illégales ou informelles. Mais cela ne suffit pas pour en tirer des conclusions convaincantes. II faut essayer de comprendre pourquoi les habitants de ces milliers de villages égyptiens, qui sont loin de manquer de savoir-faire en termes d'urbanisme et d'aménagement de l'espace, agissent comme s'ils ignoraient totalement les dangers auxquels ils s'exposent.

40 Ce sont des contraintes géographiques, économiques et/ou politiques qui se cachent le plus souvent derrière l'incohérence apparente de l'espace produit. Dans un pays où la croissance démographique impose une extension continue de l'espace habité, le développement des villes et des villages est une nécessité. Mais quand il s'agit d'un pays où la surface cultivée est trop réduite pour autoriser de nouvelles constructions, où l'espace constructible est limité par la difficulté de l'accès à l'eau, où l'ensemble des infrastructures qui autorisent une installation permanente sont quasi exclusivement regroupées le long de l'unique source d'eau et où, enfin, la proximité des champs est la condition sine qua non pour que les paysans puissent continuer à y travailler, l'espace produit par les hommes traduit inévitablement l'ensemble de ces contraintes. Si l'agglomération « déborde » sur des espaces non constructibles, ce n'est donc pas parce que les habitants « ignorent les normes ».

41 Le débat sur l'habitat informel, qui dépasse, du reste, l'Égypte et les seuls pays du tiersmonde, n'a pas manqué de ressurgir lors des dernières inondations. Il s'agit cependant de faire la part des choses entre, d'un côté, l'occupation de zones inondables - dont l'habitat informel n'est pas le seul responsable, puisque l'État lui-même n'hésite pas à occuper ces espaces - et, d'autre part, la lutte contre ce type d'habitat, qui pose notamment la question de savoir si l'État est en mesure de répondre aux besoins réels de logement.

Les secours : trop peu, trop tard

42 Aux fautes lourdes qui sont à l'origine du drame, il faut ajouter les défaillances dans l'organisation des secours. Tous les témoignages recueillis par la presse, y compris "gouvernementale ", ont insisté sur l'absence totale de secours pendant les premières heures: "Les autorités ont pris du retard pour intervenir car elles n'ont pris conscience de la gravité de la situation qu'au bout de plusieurs heures. ${ }^{29}$ "Les opérations de secours se déroulent de façon archaïque, ce qui laisse craindre l'apparition d'épidémies. $»^{30}$ «Si le premier ministre s'est rendu à Durunka dès le lendemain de la catastrophe, l'État a mis un certain temps à réagir efficacement. $»^{31}$ « Dix jours après la catastrophe de Durunka, un grand nombre d'habitants sont encore sans abri et se battent contre la maladie, le froid et la faim. Ceux qui ont été relogés dans les tentes sont entassés, femmes et hommes ensemble, et plusieurs n'ont de vêtements que le strict nécessaire pour protéger leur intimité. $\|^{32}$

43 En revanche, comme lors du tremblement de terre qui avait frappé Le Caire en octobre 1992, des organisations à sensibilité islamiste (Frères musulmans ou autres) se sont distingués par la rapidité de leurs interventions et l'efficacité de leurs secours: «Le premier ministre a bien promis aux familles une somme de 500 £e par blessé et $25 \mathrm{£e}$ 
pour chaque sans abri, mais l'urgence était le relogement de ceux qui avaient perdu leur toit. Ils ont trouvé refuge dans les mosquées d'Asyut, qui leur ont largement ouvert leurs portes. $»^{33}$ «Les habitants de Durunka critiquent le manque d'empressement des partis politiques et des autorités locales et nationales pour leur venir en aide, et insistent sur le fait que les seuls qui soient arrivés très rapidement sur les lieux étaient les Frères musulmans, qui ont commencé par distribuer de la nourriture, des vêtements et des couvertures. Aux abords d'Asyut, ils ont reçu les habitants qui fuyaient la catastrophe. $»^{34}$

Le gouvernement égyptien s'est également vu reprocher sa lenteur et son inefficacité dans les autres régions touchées par les inondations. Le plus accablant est certainement l'aveu-témoignage paru dans al-Ahrâm: «Plusieurs responsables régionaux, de peur d'être sanctionnés, ont préféré taire, dans un premier temps, la gravité de la situation, retardant ainsi les secours. ${ }^{35}$ Le plus virulent est le journal al-Sha'b (organe du Parti du Travail, à tendance islamiste) : " Nous sommes allés dans le village sinistré de Zawiya, cinq jours après la catastrophe : aucun responsable ne s'y était encore rendu, pas même le gouverneur. Pourtant, les inondations ont endommagé toutes les maisons du village. Les animaux morts se décomposent sur place et l'on craint maintenant l'apparition d'épidémies. Est-ce avec de tels responsables que nous pouvons faire face aux catastrophes naturelles ? ${ }^{36}$ De son côté, le journal al-Ahrâr (Libéraux) n'hésite pas à mettre en cause l'honnêteté même de certaines autorités: "Selon des rapports officiels, les aides aux sinistrés s'accumulent et les autorités n'ont toujours pas l'intention de les distribuer ». Le lendemain, le journal assure que les aides reçues des donateurs étrangers et nationaux ne seront pas distribuées et que «le gouvernement s'est contenté de distribuer le restant des aides reçues lors du tremblement de terre de $1992 »{ }^{37}$

Face à ces critiques, les autorités ne sont pas restées muettes. Le président Moubarak s'est rendu en personne dans les zones sinistrées et les médias officiels ont largement rendu compte de ce déplacement, comme de ceux d'autres membres du gouvernement. Mais quelques témoignages mettent en cause l'aspect relativement dérisoire de l'aide apportée aux populations sinistrées. Si le premier ministre a très rapidement décidé d'accorder 500 £e "à chaque famille ayant perdu un de ses membres » ${ }^{38}$, cette aide est venue trop tard pour discréditer les plaintes rapportées par la presse. Quant aux propos du gouverneur de Suhag, qui a assuré qu'on ne déplorait aucun manque de médicaments et que, dans chaque camp d'hébergement, il existait un centre de soins ${ }^{39}$, ils contredisent les informations rapportées par la presse $-\mathrm{y}$ compris « gouvernementale»-, qui relate largement les manques dans ce domaine ${ }^{40}$.

L'absence de politique de protection contre les risques naturels

Gamal As'ad, chroniqueur au Journal al-Sha'b, interroge les autorités :

Avez-vous entendu parler du séisme de 1992 ? Ne nous aviez-vous pas dit que vous aviez constitué une commission chargée de prévenir les catastrophes naturelles? Si cela ne vous était pas scientifiquement possible, qu'avez-vous fait pour réduire les conséquences des catastrophes naturelles? Qu'avez-vous fait pour réduire les pertes qu'elles engendrent? Le gouvernement a-t-il été surpris par les inondations? A-t-il déjà entendu parler de ces inondations qui reviennent pourtant périodiquement dans le Sinaï ou en Haute-Égypte ? (...) Y a-t-il un gouvernement qui ne démissionne pas après avoir fait preuve d'un tel laisser-aller, qui a été à l'origine de la disparition de plusieurs centaines de personnes ? ${ }^{41}$ 
47 Par ces questions et au-delà des enjeux politiques immédiats qui s'y rattachent, Gamal As'ad pointe les lacunes des systèmes de protection contre les catastrophes naturelles. «La Haute-Égypte n'avait pas connu de pluies comparables depuis 1974 ; c'est la raison pour laquelle rien n'a été fait pour limiter les conséquences de telles intempéries ", a déclaré 'Abd al-Mun'im 'Abd al-Rahmân, de la Direction de la météorologie ${ }^{42}$. La défaillance de l'État a été bien entendu exploitée par ses adversaires. Pour les Frères musulmans ou pour d'autres groupes de la mouvance islamiste, capables d'intervenir rapidement et efficacement grâce à la souplesse de leurs structures, les catastrophes naturelles sont l'occasion d'étendre un peu plus leur influence dans les couches populaires. Une opportunité pour donner corps au slogan global qui voudrait persuader les populations que, face à leurs divers problèmes et malheurs, l'islam est «la » solution. Ainsi, selon Mahmûd Habîb, responsable des Frères musulmans à Asyut, « la catastrophe est le résultat indirect de la politique de l'État dans cette ville, le seul objectif du pouvoir étant de combattre les islamistes. Le budget est consacré à l'achat d'armements alors que cette province, la plus pauvre d'Égypte, est privée de tout plan de développement $»^{43}$.

Par ailleurs, tout en accusant implicitement le gouvernement de corruption, le journal al-Sha'b n'hésite pas à demander les comptes des compensations prévues pour les sinistrés du tremblement de terre de 1992, laissant clairement entendre qu'elles ont été détournées. «Personne ne connaît le chiffre exact ni la nature des dépenses; certains disent que le gouvernement a gagné de l'argent grâce au tremblement de terre ", écrit le journal avant d'ajouter que "par son comportement, le gouvernement laisse supposer qu'il voudrait gagner de l'argent avec ces inondations $»^{44}$.

«Catastrophes naturelles ou messages divins? » C'est sous ce titre évocateur qu'al-Sha'b aborde ensuite les causes de la catastrophe : «Rien ne peut avoir lieu sans la volonté de Dieu. Les catastrophes que nous avons connues sont le message et le châtiment qui nous sont destinés. C'est un châtiment pour ce qui se fait dans ce pays et qui provoque la colère de Dieu, c'est un avertissement pour que l'on se réveille et qu'on revienne à Dieu :

- nous n'avons pas respecté la Loi divine et ses hudûd ${ }^{45}$;

- nous avons autorisé le vol et la corruption. L'argent public est gaspillé, des projets de constructions abandonnés. Les pauvres sont oubliés alors que nous dépensons des millions pour le prestige ;

- nous avons laissé couler le sang des combattants de l'islam ;

- nous avons oublié l'obligation du jihâd contre l'ennemi sioniste, le violeur de la Sainte Palestine. Nous avons dit que la guerre de 1973 était la dernière, alors que le jihâd est une obligation éternelle ;

- nous avons utilisé la télévision et le cinéma pour répandre tout ce qui s'attaque aux bases de notre morale et de notre culture ;

- nous avons limité les droits des hommes et les avons privés de la liberté d'expression et de la démocratie... $»^{46}$

50 Classique, ce genre de discours n'en trouve pas moins des échos chez ses destinataires, parmi lesquels les futurs militants de l'islamisme actif.

Une nouvelle orientation de l'aménagement du territoire?

51 Au-delà des polémiques et des stratégies, l'Égypte est confrontée à des questions essentielles: va-t-on voir apparaître une prise en compte progressive mais systématique des risques naturels dans les politiques d'aménagement du territoire? Ou 
les catastrophes naturelles, même les plus modestes, vont-elles continuer à agir comme une fatalité sur les hommes et les espaces? L'État égyptien entreprendra-t-il une politique visant à secourir efficacement les populations? Verra-t-on les nouveaux aménagements intégrer les leçons des catastrophes vécues?

Déjà, les responsables envisagent de reconstruire les villages sinistrés dans des zones moins exposées. Il est question de réinstaller les populations des villages entièrement détruits dans des zones désertiques éloignées des terres agricoles ${ }^{47}$. A court terme, d'après les déclarations officielles, 113 localités de Haute-Égypte seront concernées ${ }^{48}$.

Mais cette nouvelle politique ne peut être envisagée d'une manière autoritaire, et seule une concertation avec les populations concernées est susceptible d'éviter l'échec. Comment empêcher, en effet, de nouvelles constructions en zone inondable? Alors que Muhammad Ibrâhîm Sulayman, ministre de la Construction, explique que le nouveau site choisi pour Durunka, à deux kilomètres du village actuel, bénéficie d'une protection naturelle contre toute menace future ${ }^{49}$, les rescapés de l'ancien village refusent l'emplacement proposé, et ce pour «au moins deux raisons importantes: l'éloignement de leurs terres agricoles, et la conviction que l'ancien site était moins exposé aux «risques naturels " qu'aux accidents liés à la présence de réservoirs de pétrole. Un autre village, Nag'a Ammar, fait l'objet d'un bras de fer entre la population et les autorités: le site proposé serait infesté de fourmis blanches qui s'attaquent aux récoltes $»^{50}$.

54 Cette réticence des populations à accepter de se déplacer n'est pas récente et s'explique par leur attachement vital à la terre. Ces villages, pour une grande part, n'étaient au départ que le regroupement de maisons d'ouvriers agricoles dans les grandes 'izba (exploitations agricoles) des anciens propriétaires fonciers. L'accès à la propriété de la terre par la réforme agraire des années 50 a établi un lien puissant entre le fellah et ses parcelles. La demande de proximité n'est d'ailleurs pas uniquement affective : elle est nécessaire à l'entretien permanent des parcelles et leur irrigation continue. Ainsi, pendant vingt ans, les habitants des villages de Shaykh Tama et de Barsha (gouvernorat de Minya), gravement touchés par les inondations de 1975, ont refusé de se déplacer. Il a fallu que ces deux localités soient presque entièrement ravagées par les dernières inondations pour qu'ils acceptent d'aller vivre dans de nouveaux villages, loin des zones inondables ${ }^{51}$.

55 Dans le même gouvernorat, les habitants de Barash et Dayr al-Barasha se sont opposés à la destruction, par les forces de l'ordre, de leurs habitations, construites illégalement et en zone inondable, et se sont engagés par écrit à assumer seuls les conséquences d'éventuelles inondations. Le même phénomène s'est produit à Bani-Wasal (gouvernorat de Suhag) ${ }^{52}$.

56 Les autorités régionales se limitent donc à réfuter toute responsabilité quant aux conséquences d'inondations éventuelles sous prétexte que «les citoyens construisent dans les zones à risques et ne respectent pas les avertissements des services compétents ${ }^{53}$.

57 Le gouverneur de Suhag, accusé par la presse (gouvernementale et d'opposition) d'avoir aménagé une zone industrielle sur un terrain inondable, a déclaré que «la décision du Ministère des Ressources hydrauliques de protéger les terres inondables pouvait nuire au développement de la zone industrielle » en question ${ }^{54}$. 

devant l'impossibilité de déplacer tous les villages exposés, il importe d'appliquer systématiquement une politique de protection rapprochée reposant sur l'obligation de ne former aucun obstacle à l'écoulement des eaux des collines qui bordent la vallée et le cours du Nil. Ces règles sont d'autant plus faciles à mettre en œuvre qu'elles ne sont nullement ignorées des Égyptiens, qu'ils soient paysans, aménageurs ou hommes politiques. Par ailleurs, comme le rappelle le journal al-Ahrâm, il existe au moins deux moyens de lutter contre les torrents :

- construire le long de l'oued de petits barrages permettant une retenue d'eau utilisable pour la consommation agricole ou domestique ; ainsi réparties sur les barrages qu'elles rencontrent, les eaux perdent de leur force et arrivent affaiblies dans le fond de la vallée ;

- creuser un canal suffisamment grand et bien entretenu pour dégager vers le Nil les eaux excédentaires ${ }^{55}$, ce qui pourrait faciliter le stockage de celles-ci dans des citernes creusées dans le sol pour une utilisation ultérieure ${ }^{56}$. De son côté, le rapport du comité d'enquête du syndicat des ingénieurs a formulé un certain nombre de propositions pouvant être adoptées dans l'immédiat : « le détournement des ravins d'écoulement vers le Nil ou vers les canaux d'irrigations et de drainage ; l'étude des oueds asséchés en s'appuyant sur l'observation des inondations connues ; l'augmentation du budget du Ministère des Ressources hydrauliques pour qu'il puisse construire une infrastructure de protection contre les inondations. $\aleph^{57}$ Conclusion

Aucune communauté humaine ne peut espérer se prémunir de façon absolue contre les catastrophes dites «naturelles». Mais quand, aux caprices des dieux, s'ajoutent les contraintes économiques, sociales ou politiques, les conséquences dramatiques peuvent s'en trouver décuplées.

Si les sociétés produisent et façonnent leurs espaces, le degré de protection de ces espaces dépend directement des moyens dont elles disposent. L'empreinte des catastrophes naturelles sur une société et son espace témoigne des politiques et des moyens technologiques mis en œuvre par les véritables détenteurs du pouvoir, pour un aménagement du territoire au service de l'ensemble de la communauté. Les inondations ne tombent pas toutes du ciel...

\section{NOTES}

1. Al-Gumhuriyya, $13 / 10 / 94$.

2. Al-Ahrâm, 03/11/94.

3. Durand Dastes F., «Les mécanismes des sécheresses », Hérodote ${ }^{\circ}$ 39, p.121.

4. Al-Gumhuriyya, 16/11/94. Ces villages sont de nouveau ravagés par les torrents.

5. Al-Gumhuriyya, 18/11/94.

6. Al-Ahrâr, 21/11/94.

7. Al-Ahrâr, 15/11/94.

8. Idem.

9. Al-Gumhuriyya, 13/10/94. 
10. Idem.

11. Al-Arham Hebdo, 09-15/11/94.

12. Al-Gumhuriyya, 16/11/94.

13. Al-Ahrâr, op.cit.

14. Al-Gumhuriyya, 24/11/94.

15. Le débit se calculant en multipliant la vitesse par la section, plus la section est réduite, plus la vitesse est grande.

16. « II est clair que ces réservoirs ont été construits sur le cours des oueds en 1969. » Compte-rendu du rapport du comité technique du Syndicat des ingénieurs. al-Wafd, $15 / 11 / 94$.

17. « Lors des guerres avec Israël, l'armée a construit les réservoirs de pétrole au pied de la falaise pour les camoufler. Après la signature des accords de Camp David, ces réservoirs ont été revendus à une coopérative de carburants. Certains oléoducs en provenance de Suez traversaient le village et ont explosé lors de la catastrophe. Les habitants avaient demandé à ce que ces dépôts soient déplacés. » (Muhammad 'Abd al-Muhsin, responsable local du Parti national démocrate, alAhram Hebdo, 09-15/11/94).

18. Si l'on suppose qu'un certain nombre des habitants de ce village ont émigré - à Assiout ou vers d'autres villes -, ce doublement pourrait être plus faible que le taux de croissance naturel. 19. «Le réservoir $n^{\circ} 3$ a explosé sous l'effet d'une chute de pierres ou de la foudre, ce qui a provoqué l'incendie et l'écoulement du pétrole. Cela a entraîné l'explosion des réservoirs $n^{\circ} 6,7$ et 8. Les torrents ont entraîné le pétrole enflammé vers les maisons attenant au dépôt. »Compte rendu du rapport du comité technique du Syndicat des ingénieurs, al-Wafd, op. cit.

20. Al-Ahrâm, 04/11/94.

21. Al-Ahrâm, 12/11/94.

22. Al-Sha'b, 15/11/94.

23. Al-Ahrâm, op. cit.

24. Al-Ahram Hebdo, op.cit.

25. Al-Ahrâm, op. cit.

26. Idem.

27. Compte rendu du rapport du comité technique du Syndicat des ingénieurs, al-Wafd, op. cit.

28. Al-Ahrâm, 07/11/94.

29. Al-Ahrâm, 15/11/94.

30. Compte rendu du rapport du comité technique du Syndicat des ingénieurs : al-Wafd, op. cit.

31. Al-Ahram Hebdo, op. cit.

32. Al-Ahrâr,13/11/94.

33. Al-Ahram Hebdo, op. cit.

34. Sabah al-Khayr, 10/11/94.

35. Al-Ahrâm, op. cit.

36. Al-Sha'b, op. cit.

37. Al-Ahrâr, 14 et 15/11/94.

38. Al-Ahrâm, 07/11/94.

39. Al-Gumhuriyya, 16/11/94.

40. Idem.

41. Al-Sha'b, op. cit.

42. Al-Ahrâr, 15/11/94.

43. Al-Ahrâm Hebdo, op. cit. 
44. Al-Sha'b, op. cit.

45. Hudûd : châtiments légaux prévus par la sharîa (loi islamique).

46. Al-Sha'b, op. cit.

47. Al-Gumhuriyya, op. cit.

48. Al-Ahram Hebdo, 16-22/11/94.

49. Al-Ahram Hebdo, idem.

50. Al-Gumhuriyya, op. cit.

51. Al-Gumhuriyya, 21/11/94.

52. Al-Gumhuriyya, 24/11/94.

53. Al-Ahrâr, op. cit.

54. Al-Gumhuriyya, op. cit.

55. Al-Ahrâm, 11/11/94.

56. Al-Ahrâm, idem.

57. Al-Wafd, op. cit.

INDEX

Mots-clés : infrastructures, inondations, météo 